\title{
SERPÚLIDOS (ANNELIDA, SERPULIDAE) COLECTADOS EN LAS CAMPAÑAS OCEANOGRÁFICAS DE FAUNA IBÉRICA
}

\author{
María José Martínez \& Guillermo San Martín² \\ 'Departamento de Biología (Zoología), Centro de Investigación en Biodiversidad y Cambio global (CIBC-UAM), Facultad de \\ Ciencias, Universidad Autónoma de Madrid, calle Darwin, 2, Canto Blanco, 28049 Madrid, España. https://orcid.org/0000- \\ 0002-0467-0998 - mariajose.martinezm@estudiante.uam.es \\ ${ }^{2}$ Departamento de Biología (Zoología), Centro de Investigación en Biodiversidad y Cambio global (CIBC-UAM), Facultad de \\ Ciencias, Universidad Autónoma de Madrid, calle Darwin, 2, Canto Blanco, 28049 Madrid, España. https:// \\ orcid.org/0000-0002-8360-6221 - guillermo.sanmartin@uam.es
}

\section{RESUMEN}

Se han identificado las colecciones de poliquetos de la familia Serpulidae (Annelida) recolectados en las campañas oceanográficas de Fauna lbérica, las cuales se encuentran depositadas en el Museo Nacional de Ciencias Naturales de Madrid (MNCN), en el marco del Proyecto Fauna lbérica. Se estudiaron algo más de 100 muestras, que fueron recolectadas en el ámbito íbero-balear desde profundidades someras hasta más de $700 \mathrm{~m}$ de profundidad. Se han identificado un total de 24 especies pertenecientes a 15 géneros; se han observado algunas anomalías morfológicas, como por ejemplo características intermedias entre dos especies de Serpula o quimeras bioperculadas en Hydroides. También se ha profundizado en el estudio de los complejos de géneros Apomatus-Protula y Filograna-Salmacina.

Palabras clave: Serpulidae; Polychaeta; Annelida; área íbero-balear.

\section{ABSTRACT}

Serpulids (Annelida, Serpulidae) collected in the oceanographic campaigns of Fauna Ibérica

The collections of polychaetes of the family Serpulidae (Annelida) collected in the oceanographic campaigns of "Fauna Ibérica" have been identified, which are deposited in the National Museum of Natural Sciences of Madrid (MNCN), within the framework of the Iberian Fauna Project. More than 100 samples were studied, which were collected in the Iberian-Balearic area from shallow depths to more than $700 \mathrm{~m}$ depth. A total of 24 species belonging to 15 genera have been identified; some morphological anomalies have been observed, for example, intermediate characteristics between two species of Serpula or bioperculated chimeras in Hydroides. The study of the complexes of genera Apomatus-Protula and Filograna-Salmacina has also been studied.

Keywords: Serpulidae; Polychaeta; Annelida; Iberian-Balearic area.

Recibido/Received: 28/09/2018; Aceptado/Accepted: 12/12/2018; Publicado en línea/Published online:25/02/2019

Cómo citar este artículo/Citation: Martínez, M. J. \& San Martín, G. 2019. Serpúlidos (Annelida, Serpulidae) colectados en las campañas oceanográficas de Fauna Ibérica. Graellsia, 75(1): e085. https://doi.org/10.3989/graellsia.2019.v75.224

Copyright: (c) 2019 SAM \& CSIC. This is an open-access article distributed under the terms of the Creative Commons Attribution 4.0 International (CC BY 4.0) License. 


\section{Introducción}

Los serpúlidos, familia Serpulidae Rafinesque, 1815 , son una familia de poliquetos sésiles, la mayoría marinos, que viven en el interior de tubos calcáreos que ellos mismos fabrican con las glándulas del collar (Zibrowius, 1968). Dichos tubos suelen encontrarse adheridos a sustratos duros, a excepción de las especies del género Ditrupa Berkeley, 1835, cuyos tubos se encuentran libres sobre sustratos blandos. Se caracterizan por su metastomio segmentado y regionalizado en tórax y abdomen, su penacho branquial y, en una buena mayoría de especies, la presencia de un opérculo (en ocasiones más de uno); el tamaño de los ejemplares varía según las especies, desde unos pocos milímetros hasta $25 \mathrm{~cm}$ en algunas especies de Protula Risso, 1826. Se trata de una familia diversa, ampliamente distribuida en el medio marino, aunque también hay especies que habitan los ambientes de estuarios y, excepcionalmente, ambientes de agua dulce en cuevas.

Junto con los sabélidos, familia Sabellidae Latreille, 1825 , son conocidos como "gusanos-plumero" por sus coloridos penachos branquiales o radiolas que asoman de sus tubos. Los serpúlidos son un grupo abundante y relativamente fácil de identificar, salvo algunos casos concretos, existiendo en la bibliografía especializada numerosas citas para las costas íbero-baleares. Recientemente, Alcázar \& San Martín (2016) han publicado un catálogo actualizado de esta familia para el ámbito íbero-balear. La familia en el ámbito mundial consta de unas 500 a 600 especies (BastidaZavala, 2009), distribuidas en unos 70 géneros válidos, de los cuales 46 se incluyen en la subfamilia Serpulinae Rafinesque, 1815 y unos 24 en Spirorbinae Chamberlin, 1919 (ten Hove \& Kupriyanova, 2009). Hay aún muchos géneros que necesitan una revisión, por lo que el número real de especies conocidas es todavía incierto. En el ámbito íbero-balear se han registrado 60 especies, pertenecientes a 28 géneros.

De acuerdo con ten Hove (1984), Fitzhugh (1989), Bastida-Zavala (2009) y ten Hove \& Kupriyanova (2009), los serpúlidos constituyen un grupo monofilético cuyas sinapomorfías que los definen son la existencia de membrana torácica y tubo calcáreo (Rouse \& Pleijel, 2001). Rouse \& Fauchald (1997) los sitúan, junto con los sabélidos y otras familias, en el orden Sabellida, que pertenece al clado Canalipalpata Rouse \& Fauchald, 1997 no Linnaeus (Polychaeta Grube, 1850) (Rouse, 2000). Los sabélidos constituyen el grupo hermano de los serpúlidos, con los que comparten el carácter de la inversión setal, es decir que en el tórax las sedas son dorsales y los uncinos ventrales, mientras que en el abdomen sucede al revés. Esta característica está algo enmascarada en los Spirorbinae, debido al giro de $90^{\circ}$ en su cuerpo y tubo.

Aunque la familia se considera bien definida, existe controversia sobre su clasificación en subfamilias. La más clásica es la que divide los serpúlidos en cuatro subfamilias: Serpulinae, Spirorbinae, Filograninae Rioja, 1923 y Ficopomatinae Pillai, 1960. La primera división de la familia la realizó Chamberlin (1919), en la que establece dos grandes subfamilias: Serpulinae (con el cuerpo simétrico) y Spirorbinae (con el cuerpo asimétrico y el tubo espiralizado). Posteriormente, Rioja (1923) erigió la subfamilia Filograninae para aquellos serpúlidos sin opérculo, y Pillai (1960) define la subfamilia Ficopomatinae para aquellos serpúlidos que viven en aguas salobres y tienen las sedas del collar aserradas.

No se ha realizado ningún análisis cladístico que incluya todos los géneros de serpúlidos, ni se ha comprobado la validez de las distintas subfamilias propuestas, utilizando una metodología objetiva (Bastida-Zavala, 2009). Por este motivo, y siguiendo a ten Hove \& Kupriyanova (2009), en este trabajo se considera a los serpúlidos como una familia válida, tratando a los espirorbinos como una subfamilia, ya que es la única homogénea y monofilética, dejando a todos los demás serpúlidos dentro de la subfamilia Serpulinae, aunque conscientes de su parafilia.

\section{Material y métodos}

El material que se ha identificado procede mayoritariamente de la campaña oceanográfica Fauna Ibérica III, aunque también hay material de las campañas I, II y IV. En el Apéndice se facilitan los datos de interés de dicho material. Se realizaron cuatro campañas oceanográficas que abarcan la mayor parte del ámbito íbero-balear a bordo del buque Oceanográfico García del Cid, del Consejo Superior de Investigaciones Científicas. El material estudiado se recolectó mediante arrastre bentónico y buceo autónomo. En la primera campaña, dentro del proyecto Fauna Ibérica I (1989), se tomaron muestras principalmente entre 60 - $500 \mathrm{~m}$ de profundidad en el golfo de Cádiz, a ambos lados del estrecho de Gibraltar, mar de Alborán y bajos de Motril; tratándose de una zona de especial interés por la confluencia de hasta tres regiones biogeográficas (Lusitana, Mauritana y Mediterránea). En la segunda campaña (1994) se muestrearon las costas cantábricas, desde las islas Cíes (Pontevedra) hasta las costas orientales de Guipúzcoa y el banco de Galicia en el rango de profundidad entre 30 - $770 \mathrm{~m}$. La siguiente campaña se realizó en 1994 durante el proyecto Fauna Ibérica III en las islas Baleares y banco del Emile Baudot (sureste de Baleares) y, de forma muy puntual, se muestrearon los islotes Columbretes (Castellón), el rango batimétrico osciló entre 0-200 m. La última campaña se desarrolló en el marco del proyecto Fauna Ibérica IV, en 1996; las zonas prospectadas fueron las aguas de los islotes Columbretes, mar de Alborán e islas Hormigas (Murcia), siendo los rangos batimétricos entre 0-500 m.

Todo el material de poliquetos recolectado se separó por familias en los laboratorios del Museo Nacional 
de Ciencias Naturales de Madrid (MNCN). Los ejemplares se fijaron con formol al $5 \%$ y se preservaron en etanol al $70 \%$.

Los ejemplares se han identificado utilizando la clave de géneros y especies ibéricas de serpúlidos de Fauna Ibérica (San Martín, en preparación), así como otros trabajos previos, como los de Zibrowius (1968) y Bianchi (1981).

\section{Resultados}

Se han estudiado un total de 640 ejemplares, de los cuales 534 se han identificado a nivel específico, el resto no han podido ser determinados. Se identificaron 24 especies pertenecientes a 15 géneros, que suponen el $39 \%$ de las especies y el $52 \%$ de los géneros de serpúlidos ibéricos conocidos. Respecto al número de ejemplares, se debe señalar que muchas muestras identificadas como Salmacina Claparède, 1869 forman agregados de cientos de tubos, siendo difícil cuantificar el número de ejemplares exactos que presenta la muestra. En estos casos solo se ha tenido en cuenta el número de ejemplares separados de sus tubos y estudiados.

El listado de géneros y especies se ordena alfabéticamente, añadiendo para cada especie una referencia de la localidad (ver Apéndice) y entre paréntesis el número de ejemplares estudiados.

Algunos ejemplares solo se han podido identificar a nivel de género, debido a que no se han podido observar caracteres fundamentales para la identificación a nivel específico, no incluyéndolos en la lista taxonómica.

\section{Género Apomatus Philippi, 1844}

\section{Apomatus ampulliferus Philippi, 1844}

Estaciones y material examinado: 250A (3), 317A (2), 316A (2)

Distribución íbero-balear: Citado de la bahía de Palma de Mallorca y cabo Vilán (A Coruña) (Alcázar \& San Martín, 2016); isla de Alborán (Almería) y cabo Berbería (isla Formentera).

\section{Género Ditrupa Berkeley, 1835}

\section{Ditrupa arietina (Müller, 1776)}

Estaciones y material examinado: 192A (1), 240B4 (5), 194A (1)

Distribución íbero-balear: Ha sido citada en el Cantábrico (Ondarroa, Ribadesella, cabo de Peñas); Atlántico (Galicia, varias localidades de Portugal); Mediterráneo (isla de Alborán, Valencia, Mallorca, islas Columbretes y Cataluña) (Gil, 2011; Alcázar \& San Martín, 2016).
Género Filograna Berkeley, 1835

Filograna implexa Berkeley, 1835

Estaciones y material examinado: 191B5 (21), 218B (10), 192A (2), 282B15 (1), 281B14 (1), 240B (1)

Distribución íbero-balear: Se ha citado en Berlenga, costa de Arrábida, Algarve, Sesimbra (Portugal) (Gil, 2011); Santander, San Vicente de la Barquera (Cantabria), Fornelos, Batel y Segaño (Galicia), Gibraltar, mar de Alborán, islas Chafarinas (Melilla), Valencia, Baleares, islas Columbretes, islas Medas (Gerona), cabo de Creus (Mediterráneo) (Alcázar \& San Martín, 2016).

\section{Género Filogranula Langerhans, 1884}

\section{Filogranula calyculata (Costa, 1861)}

Estaciones y material examinado: 317A (1)

Distribución íbero-balear: Ha sido citada del cabo San Vicente y cabo Espichel (Portugal), Cantabria y Asturias (Gil, 2011; Alcázar \& San Martín, 2016); isla de Alborán (Almería).

\section{Género Hyalopomatus Marenzeller, 1878}

\section{Hyalopomatus marenzelleri Langerhans, 1884}

Estaciones y material examinado: 248A (4)

Distribución íbero-balear: Ha sido citada en el cañón de Setúbal (Portugal) y banco de Galicia (Gil, 2011; Alcázar \& San Martín, 2016); isla de Formentera (Baleares).

\section{Género Hydroides Gunnerus, 1768}

\section{Hydroides niger Zibrowius, 1971}

Estaciones y material examinado: 179B2 (1)

Distribución íbero-balear: Especie típicamente mediterránea, rara o ausente en los sectores septentrionales, aunque presente en el Adriático (Bianchi, 1981). Ha sido citada en Nerja (Málaga), Alborán, Almería, islas Chafarinas, Murcia y Baleares. También en el País Vasco, y en el Algarve (Portugal), con dudas.

\section{Hydroides norvegicus Gunnerus, 1768}

Estaciones y material examinado: 248A (1), 106A (3), 152A (10), 142A (4), 192A (4), 323A (1), 151A (1), 105A (3), 111A (7), 312A (2), 305A (1), 169A (10), 153A (3), 150A (6), 107A (6), 91A (9), 266A (1), 168A (3), 119A (5), 114A (4), 322A (1), 315B2 (1), 268A(1), 101A (1), 283A (3), 102A (1), 238A (1), 216A (2), 109A (8), 148A (4), 113A (1)

Observaciones: Las muestras 106A, 192A, 153A, 283A, 238A, 113A presentan ejemplares bioperculados. 
Distribución íbero-balear: Ha sido citada en localidades que prácticamente cubren todo el ámbito ibérico y balear (Gil, 2011; Alcázar \& San Martín, 2016).

\section{Hydroides pseudouncinatus Zibrowius, 1968}

Estaciones y material examinado: 263B2 (1), 218B10 (1)

Distribución íbero-balear: Está citada en Coruña y numerosas localidades a lo largo de todo el litoral mediterráneo (Alcázar \& San Martín, 2016).

Género Janita Saint-Joseph, 1894

Janita fimbriata Delle Chiaje, 1822

Estaciones y material examinado: 316A(1), 179B3 (1)

Distribución íbero-balear: Citada en San Sebastián, Santander, Gijón, Alborán, varios puntos de Andalucía, Baleares y cabo de Creus y otras localidades catalanas (Alcázar \& San Martín, 2016).

\section{Género Placostegus Philippi, 1844}

Placostegus crystallinus (non Scacchi, 1836) sensu Zibrowius, 1968

Estaciones y material examinado: 267B4 (1)

Distribución íbero-balear: Ha sido citada en Galicia, Baleares, y localidades de Gerona (Alcázar \& San Martín, 2016); islas Columbretes (Castellón).

\section{Placostegus tridentatus (Fabricius, 1779)}

Estaciones y material examinado: 235A (4), 203B1 (1), 216A (4), 202A (1), 248A (4)

Distribución íbero-balear: Ha sido citada en Cantabria, Asturias, Portugal, mar de Alborán, Valencia, Mallorca, Menorca, Castellón, golfo de Rosas y otras localidades catalanas (Alcázar \& San Martín, 2016).

Género Pomatoceros Philippi, 1844

\section{Pomatoceros lamarcki (Quatrefages, 1866)}

Estaciones y material examinado: 200A (1)

Distribución íbero-balear: Citada en numerosas localidades cantábricas, en Portugal y a lo largo de toda la costa mediterránea española (Alcázar \& San Martín, 2016).

\section{Pomatoceros triqueter (Linnaeus, 1758)}

Estaciones y material examinado: 183B5 (1), 277B15 (1), 322A (1), 276A (2), 236B4 (1), 281B11 (2), 281B3 (1)

Distribución íbero-balear: Citada en numerosas ocasiones a lo largo de todo el ámbito ibérico y balear (Alcázar \& San Martín, 2016).

\section{Género Protula Risso, 1826}

Protula intestinum (Lamarck, 1818)

Estaciones y material examinado: 145A (1), 313A (1), 275B2 (1), 317A (7), 238A (13), 266A (1), 323A (2), 275B9 (1), 315B2 (1), 231A (1), 194A (1), 228A (2)

Distribución íbero-balear: Ha sido citada en toda la costa mediterránea, desde Gibraltar a Cataluña, así como Baleares (Alcázar \& San Martín, 2016).

\section{Protula tubularia (Montagu, 1803)}

Estaciones y material examinado: 285B22 (1), 277B24 (1), 275B13 (1), 322A (1)

Distribución íbero-balear: Ha sido citada en numerosas localidades a lo largo de todo el ámbito ibérico y balear (Alcázar \& San Martín, 2016).

\section{Género Salmacina Claparède, 1869}

\section{Salmacina dysteri (Huxley, 1855)}

Estaciones y material examinado: 274B5 (1), 56A (2), 238A2 (7), 134A (4), 227A (4), 270B4 (2), 168A (4), 316A (1), 285B22 (2), 302B5 (1), 285B12 (1), 281B4 (1), 239A (1), 277B19 (1), 296B10 (8), 189A (4), 182A (1), 267B2 (4), 262B2 (3), 263B2 (4), 238A (4), 218B (1), 179B2 (2), 268A (1), 266A (1), 252A (1), 113A (2)

Distribución íbero-balear: Ha sido citada en todos sus sectores, desde el País Vasco hasta Cataluña (Alcázar \& San Martín, 2016).

\section{Género Serpula Linnaeus, 1758}

Serpula concharum Langerhans, 1880

Estaciones y material examinado: 111A (3), 254B (2), 313A (1), 107A(1), 106A(1), 322A (1), 192A (1), 136A (1), 315B2 (1), 281B3 (1), 142A (2)

Distribución íbero-balear: Ha sido citada desde el País Vasco a Cataluña (Alcázar \& San Martín, 2016).

\section{Serpula lobiancoi Rioja, 1917}

Estaciones y material examinado: 239A (1), 136A (1), 213A (1), 317A (2), 158A (1), 238A (4), 252A (2)

Distribución íbero-balear: Ha sido citada en las costas de Cantabria, Asturias, Portugal, Gibraltar, Andalucía, mar de Alborán, Valencia y Gerona (Alcázar \& San Martín, 2016); islas de Formentera, Menorca e Ibiza (Baleares).

\section{Serpula vermicularis Linnaeus, 1767}

Estaciones y material examinado: 178A (1), 213A (5), 218B (1), 152A (2), 324A (4), 322A (5), 314A (1), 106A (2), 191B5 (1), 153A (4), 238A (18), 239A (11), 
266A (6), 168A (5), 316A (3), 194A (2), 263B2 (2), 216A (3), 105A (1), 281B14 (1), 250A (13), 258B3 (1), 313A (3), 323A (1), 328B (4), 293A (1), 326A (1), 311B3 (1), 295B1 (3), 305A (1), 281B3 (2), 304A (1), 158A (1), 148A (1), 352A (1), 277B15 (1), 290B9 (1), 283A (1), 189A (1), 199P (1), 190B5 (1), 248A (2), 240B10 (1), 230B14 (1), 269A (1), 192A (6), $217 \mathrm{~B} 14$ (1), 230B5 (1), 267B2 (1), 258B10 (1), 127A (3), 317A (1), 113A (7), 307A (1)

Observaciones: En numerosos ejemplares se ha observado que presentan características intermedias entre Serpula vermicularis y Serpula cavernicola Fassari y Mòllica, 1991, que pudiera atribuirse a variabilidad intraespecífica o a formas juveniles.

Distribución íbero-balear: Ha sido citada en numerosas ocasiones en todo el ámbito íbero-balear (Alcázar \& San Martin, 2016).

\section{Género Spirobranchus Blainville, 1818}

\section{Spirobranchus polytrema (Philippi, 1844)}

Estaciones y material examinado: $217 \mathrm{~B} 14$ (1), 142A (1), 258B10 (1), 223B7 (1), 230B1 (1), 179B2 (3), 179B3 (2)

Distribución íbero-balear: Ha sido citada en todas sus costas, desde el País Vasco a Cataluña y Baleares, incluyendo también Portugal (Alcázar \& San Martín, 2016).

\section{Género Vermiliopsis Saint-Joseph, 1894}

\section{Vermiliopsis infundibulum (Philippi, 1844)}

Estaciones y material examinado: 266A (1), 323A (1), 252A (1), 218B (2), 136A (1), 216A (1), 250A (1), 327A (1), 324A (1), 192A (2), 178A (1), 352A (1), 283 (1), 276A (3), 219A (2), 267B2 (2), 238A (5), 316A (1), 269A (1), 235A (1)

Distribución íbero-balear: Aparentemente cosmopolita (Gil, 2011). Citada a lo largo de todas sus costas, desde el País Vasco a Cataluña, incluyendo Portugal y Baleares.

\section{Vermiliopsis labiata (Costa, 1861)}

Estaciones y material examinado: 183B5 (1)

Distribución íbero-balear: Ha sido citada en toda la costa mediterránea (Alcázar \& San Martín, 2016).

\section{Vermiliopsis monodiscus Zibrowius, 1968}

Estaciones y material examinado: 172A (1), 173A (6)

Distribución íbero-balear: Solo se conoce de unas pocas localidades del Mediterráneo (golfo de Marsella), mar Tirreno, Grecia (Gil, 2011). Se ha citado en dos ocasiones, una en las costas de Portugal y en el mar de Alborán, aunque ambas se consideran dudosas.
Se registra por primera vez en las costas de Galicia, confirmando su presencia en la península Ibérica.

\section{Vermiliopsis striaceps (Grube, 1862)}

Estaciones y material examinado: 185B1 (1), 173A (1), 183B5 (1), 254B (4)

Distribución íbero-balear: Ha sido citada en Galicia, costa mediterránea andaluza, mar de Alborán, mar Menor, Valencia, Baleares y cabo de Creus (Alcázar \& San Martín, 2016).

\section{Discusión}

\section{Características intermedias en el género Serpula}

El género Serpula, en el ámbito íbero-balear, lo integran cuatro especies ibéricas: $S$. cavernicola, $S$. concharum, S. lobiancoi y S. vermicularis. Todas ellas, a excepción de $S$. cavernicola, se encuentran presentes entre las muestras recogidas en las campañas oceanográficas de Fauna Ibérica estudiadas en este trabajo. Estas cuatro especies difieren en aspectos relacionados con el opérculo, el tubo, los uncinos abdominales y las sedas especiales del collar.

Las especies $S$. concharum y $S$. lobiancoi se han identificado fácilmente ya que presentan opérculos y tubos muy característicos. Sin embargo, no todos los ejemplares identificados como $S$. vermicularis poseen rigurosamente los caracteres que definen la especie (Fassari \& Mòllica, 1991), sino que se han encontrado una serie de ejemplares que presentan caracteres específicos de $S$. vermicularis junto con caracteres específicos de $S$. cavernicola. Todos estos ejemplares se han identificado como $S$. vermicularis, teniendo en cuenta únicamente la morfología de las sedas del collar. Normalmente, las sedas del collar de S. vermicularis presentan dos grandes dientes basales y el borde serrado, mientras que $S$. cavernicola presenta un gran diente basal y el borde liso. Sin embargo, se han encontrado ejemplares cuyas sedas especiales del collar presentan una combinación de ambos tipos, de manera que se han encontrado sedas con dos dientes grandes en la base y el borde completamente liso. En este trabajo, solo se ha tenido en cuenta el número de dientes grandes basales y se ha obviado la morfología del borde de las sedas, de manera que los ejemplares que presentan dos grandes dientes basales, independientemente del tipo de borde, se les ha identificado como $S$. vermicularis. No se ha encontrado ningún ejemplar de Serpula cuyas sedas del collar tengan un solo diente basal grande, que son las sedas típicas de $S$. cavernicola. Las especies claramente identificadas como $S$. vermicularis presentan tubos de colores rosáceos, anaranjados, asalmonados y violáceos, además de una serie de carenas, en ocasiones, claramente denticuladas. Sin embargo, algunos ejemplares estudiados de $S$. vermicularis presentan tubos de color blanco 
y completamente lisos (a excepción de las líneas de crecimiento). Una vez más, dichos caracteres coinciden con los caracteres específicos del tubo de $S$. cavernicola; además, muchos de estos ejemplares presentan un número muy elevado de dientes marginales del opérculo (más de 45), carácter típico de $S$. cavernicola. Por lo que es importante destacar la variedad intraespecífica que se ha podido constatar en la muestra de población de Serpula vermicularis identificada en las campañas de Fauna Ibérica.

\section{Complejo de géneros Hydroides-Serpula}

El género Hydroides se compone de ocho especies ibéricas, de las cuales solo tres $(H$. niger, H. norvegicus e H. pseudouncinatus) han podido ser identificadas en el material estudiado. Las especies de este género se diferencian claramente por la morfología del opérculo y del verticilo, pero en este trabajo se ha encontrado cierta dificultad en el estudio de los opérculos de $H$. elegans Haswell, 1883 e H. norvegicus. Como apuntan Bastida-Zavala \& Salazar-Vallejo (2000), H. elegans ha sido confundida en muchas ocasiones con $H$. norvegicus, debido a que las espinas operculares son parecidas y muy variables. La confusión existente entre ambas especies fue resuelta por Zibrowius (1971) y Ten Hove (1974), quienes observaron que la diferencia principal se encuentra en la denticulación basal de las sedas del collar; $H$. norvegicus presenta dos grandes dientes en la parte media de la seda, mientras que $H$. elegans tiene muchos dentículos que le dan un aspecto piloso. Cuando $H$. norvegicus pierde el verticilo, es difícilmente distinguible de $S$. concharum porque ambas especies poseen el mismo tipo de sedas del collar. Lo único en lo que se diferencian en este caso particular es en la forma, color y sección del tubo. Esto ha podido ocurrir en un caso en el que se encontró un ejemplar juvenil de $S$. concharum entre numerosos individuos adultos de $H$. norvegicus.

\section{Quimeras bioperculadas de Hydroides norvegicus}

La "'"quimera bioperculada" es un término acuñado por ten Hove \& Ben-Eliahu (2005) para un estado ontogenético en algunas especies de Hydroides, que presentan dos opérculos de diferente tipo, uno pequeño con espinas simples indiferenciadas, presente en el lóbulo branquial opuesto al de un opérculo característico de la especie. La primera quimera bioperculada de la especie $H$. norvegicus hallada en las costas de las islas Sisargas (Galicia) fue descrita en el trabajo de Alcázar \& San Martín (2016). En este trabajo, de nuevo se han encontrado ejemplares bioperculados de la misma especie recolectados en Asturias, Baleares, Castellón, Lugo y Vizcaya.

Hydroides norvegicus es una especie con cierta tendencia a desarrollar quimeras bioperculadas (Rioja, 1919; Sentz, 1962; Alcázar \& San Martín, 2016), bien con ambos opérculos iguales o bien uno de tipo "Hydroides" y otro de tipo "Serpula". Como explican Bastida-Zavala \& Salazar-Vallejo (2000), el desarrollo del opérculo de Hydroides y su duplicidad ha llamado la atención desde hace mucho tiempo. Rioja (1919, 1944) y de León- González (1990) analizaron especies del género Hydroides del Atlántico español y del Pacífico mexicano, respectivamente. Todos los casos descritos se refieren a $H$. norvegicus, teniendo siempre verticilo el opérculo principal. Sin embargo, el segundo opérculo se ha encontrado tanto con verticilo como sin él. Se han hallado cinco ejemplares cuyos opérculos eran iguales (ambos de tipo "Hydroides") y un ejemplar cuyos opérculos eran diferentes (uno con verticilo y otro sin verticilo). Además, en este último caso el opérculo aparentemente más desarrollado era de tipo "Serpula", por lo que se han estudiado las sedas del collar para identificar el ejemplar.

\section{Complejo de géneros: Apomatus-Protis-Protula}

El género Protis Ehlers, 1887 se diferencia de los géneros Apomatus y Protula en la ausencia de sedas especiales del collar. Y los géneros Apomatus y Protula a simple vista se diferencian tan solo en la presencia o ausencia de opérculo, respectivamente. Dado que el desarrollo del opérculo en Apomatus puede no haberse producido o que el opérculo se pueda perder secundariamente, hay bastante controversia sobre la identidad de este género con respecto a Protula. Algunos autores, como ten Hove \& Pantus (1985), los consideran géneros separados debido a que se trata de una clasificación basada en un carácter morfológico variable. En cambio, autores como Kupriyanova \& Jirkov (1997) los sinonimizan. Para la identificación de estos géneros problemáticos, se ha seguido la clasificación de ten Hove \& Pantus (1985) y el carácter utilizado para su identificación ha sido la presencia (Apomatus) o ausencia (Protula) de opérculo. Como futuras líneas de investigación, se ve necesario un estudio molecular y filogenético que aporte más datos sobre la relación de estos dos géneros. Además, estudios detallados (ten Hove \& Pantus, 1985) sobre la anatomía de ambos géneros muestran una serie de caracteres que permiten diferenciarlos:

\footnotetext{
Tipo de patrón de vasos sanguíneos ventrales torácicos. El sistema circulatorio de Protula presenta vasos transversales mientras que en Apomatus carece de los mismos. Este carácter no se ha tenido en cuenta en este trabajo porque sería necesario otro tipo de estudio anatómico más profundo y no parece posible observarlo en animales conservados.

- Las manchas oculares presentes en las radiolas son diferentes en ambos géneros, Protula presenta ojos sencillos o independientes y Apomatus ojos agrupados o en línea. En este trabajo, no se han encontrado manchas oculares en todos los ejemplares de ambos géneros, cuando se han encontrado no han proporcionado una información decisiva, por lo que no se ha tenido en
} 
cuenta este carácter. Ten Hove \& Nishi (1996) explican que los ocelos en la membrana del collar, en la corona branquial y en el embudo opercular, cuando existen, se pierden con facilidad tras la fijación y conservación de los ejemplares de serpúlidos.

- Longitud de las sedas capilares y limbadas del collar. Las sedas del collar de Apomatus presentan mayor longitud que las de Protula, en el presente trabajo no se ha podido discernir esta diferencia.

- La presencia de sedas en las partes anterior y posterior del abdomen. Este carácter resulta imprescindible para la identificación a nivel de especie, de manera que los ejemplares que presentan sedas abdominales falciformes pertenecen a las especies $A$. similis Marion \& Bobretzky, 1875 o P. tubularia; y los que presentan sedas abdominales geniculadas se identifican como A. ampulliferus o $P$. intestinum.

Con esta pequeña revisión del complejo Apomatus/ Protula, en este trabajo solo se ha tenido en cuenta el carácter opérculo para distinguir con claridad los géneros Apomatus y Protula. Siendo necesario señalar la confusión existente entre $P$. tubularia y $A$. similis en los casos en los que el ejemplar haya perdido o no haya desarrollado el opérculo. Si esta situación se diera en ejemplares de $A$. ampulliferus y $P$. tubularia, ambas especies se pueden distinguir debido a que presentan diferentes tipos de sedas abdominales, geniculadas y falciformes respectivamente. Lo mismo ocurre con las especies $A$. similis y $P$. intestinum (falciformes y geniculadas, respectivamente).

Trabajos de Fauvel (1927), Bianchi (1981) y Kupriyanova (1993) mencionan otra diferencia entre ambos géneros, y es la distribución de las sedas falciformes o geniculadas del abdomen, presentando Protula sedas de este tipo a partir de la mitad del abdomen y Apomatus a lo largo de todo el abdomen.

\section{Complejo de géneros Salmacina-Filograna}

Un problema parecido al comentado con Apomatus y Protula es el que ocurre en los géneros Filograna y Salmacina. Algunos autores los han considerado sinónimos, pero Nogueira \& ten Hove (2000) hicieron un estudio exhaustivo y llegaron a la conclusión de que son dos géneros diferentes por la presencia de dos opérculos en Filograna y la ausencia de ellos en Salmacina. No se ha observado en ninguno de los ejemplares identificados de Filograna la presencia de un opérculo doble, solo se ha constatado la presencia de uno. Esto puede deberse a que ambos opérculos se encuentran fuertemente adheridos, de modo que parecen un único opérculo.

\section{Agradecimientos}

Le agradecemos al Museo Nacional de Ciencias Naturales de Madrid (MNCN) el haber facilitado el material necesario para este estudio, en especial a Javier Sánchez y Francisco Yagüe.
Así como la labor de los revisores Romana Capaccioni-Azzati y Jorge Núñez de la presente revista. Y la Universidad Autónoma de Madrid (UAM) por haber financiado dicho trabajo.

\section{Referencias}

Alcázar, J. \& San Martín, G., 2016. Serpúlidos (Annelida, Serpulidae) colectados en la campaña oceanográfica "Fauna II" y catálogo actualizado de especies iberobaleares de la familia Serpulidae. Graellsia, 72(2): 1-18. https://doi.org/10.3989/graellsia.2016.v72.120

Bastida-Zavala, J. R., 2009. Serpulidae Rafinesque, 1818. In: de León-González, J. A., Bastida-Zavala, J. R., Carrera-Parra, L. F., García-Garza, M. E., Peña-Rivera, A., Salazar-Vallejo, S. I. \& Solís-Weiss, V. (eds.). Poliquetos (Annelida: Polychaeta) de México y América Tropical. Vol. 43. Universidad Autónoma de Nuevo León. Monterrey: 521-554.

Bastida-Zavala, J. R. \& Salazar-Vallejo, S. I., 2000. Serpúlidos (Polychaeta: Serpulidae) del Caribe noroccidental: Hydroides y Serpula. Revista de Biología Tropical, 48(4): 841-858.

Bianchi, C. N., 1981. Policheti Serpuloidei. Guide per il Riconoscimento delle Specie Animali delle Acque Lagunari e Costiere Italiane, 5: 1-187.

Chamberlin, R. V., 1919. The Annelida Polychaeta (Rep. Sci. Res. Exp. "Albatross"). Memoirs of the Museum of Comparative Zoology, 48: 1-514.

Fassari, G. \& Mòllica, E., 1991. Una nuova specie di Serpulidae (Annelida Polychaeta) di grotta. Animalia, 18: 261-267.

Fauvel, P., 1927. Polychètes sédentaires. Addenda aux errantes, Archiannélides, Myzostomaires. Faune de France, 16: 1-494.

Fitzhugh, K., 1989. A systematic revision of the SabellidaeCaobangidae-Sabellongidae complex (Annelida: Polychaeta). Bulletin of the American Museum of Natural History, 192: 1-104.

Gil, J. C., 2011. The European Fauna of Annelida Polychaeta. Tese de Doutorado. Universidade de Lisboa. 1554 pp. (inédita).

Hove, H. A. ten, 1974. Notes on Hydroides elegans (Haswell, 1883) and Mercierella enigmatica Fauvel, 1923, alien serpulid polychaetes introduced into the Netherlands. Bulletin Zoölogisch Museum, 4(6): 45-51.

Hove, H. A. ten, 1984. Towards a phylogeny in serpulids (Annelida: Polychaeta). In: Hutchings, P. A. (ed.). Proceedings of the First International Polychaete Conference, Sydney, 1983. Linnean Society of New South Wales: 181-196.

Hove, H. A. ten \& Ben-Eliahu, M. N., 2005. On the identity of Hydroides priscus Pillai, 1971-Taxonomic confusion due to the ontogeny in some serpulid genera (Annelida: Polychaeta: Serpulidae). Senckenbergiana Biologica, 85(2): 127-145.

Hove, H. A. ten \& Kupriyanova, E. K., 2009. Taxonomy of Serpulidae (Annelida, Polychaeta): the state of affairs. Zootaxa, 2036: 1-126. 
Hove, H. A. ten \& Nishi, E., 1996. A redescription of the Indo-West Pacific Spirobranchus corrugatus Straughhan, 1967 (Serpulidae, Polychaeta), and an alterenative hypothesis on the nature of a group of Middle Miocene microfossils from Poland. Beaufortia, 46(5): 83-96.

Hove, H. A. ten \& Pantus, F. J. A., 1985. Distinguishing the genera Apomatus Philippi, 1844 and Protula Risso, 1826 (Polychaeta: Serpulidae). A further plea for a methodical approach to serpulid taxonomy. Zoologische Mededelingen, 59(32): 419-437.

Kupriyanova, E. K., 1993. [Deep-water Serpulidae (Annelida, Polychaeta) from Kurile-Kamchatka Trench. 2. Genera Bathyditrupa, Bathyvermilia, and Protis.] Zoologichesky Zhurnal, 72(3): 21-28. (en ruso).

Kupriyanova, E. K. \& Jirkov, I.A., 1997. Serpulidae (Annelida, Polychaeta) of the Arctic Ocean. Sarsia, 82: 203-236. https://doi.org/10.1080/00364827.1997.10413651

León-González, J. A. de, 1990. Dos serpúlidos nuevos para el Pacífico mexicano y duplicidad opercular en Hydroides crucigerus (Polychaeta: Serpulidae). Revista de Biología Tropical, 38: 335-338.

Nogueira, J. M. M. \& Hove, H. A. ten, 2000. On a new species of Salmacina Claparède, 1870 (Polychaeta: Serpulidae) from São Paulo State, Brazil. Beaufortia, 50(8): 151-161.

Pillai, T. G., 1960. Some marine and brackish-water serpulid Polychaeta from Ceylon, including new genera and species. The Ceylon Journal of Science (Biological Sciences), 3(1): 1-40.

Rioja, E., 1919. Una curiosa anomalía del "Hydroides norvegica" Gunn. y algunas consideraciones acerca de la filogenia de los serpúlidos. Boletín de la Real Sociedad Española de Historia Natural, 19: 445-449.
Rioja, E., 1923. Estudio sistemático de las especies ibéricas del suborden Sabelliformia. Trabajos del Museo Nacional de Ciencias Naturales, Serie Zoológica, 48: 5-144.

Rioja, E., 1944. Estudios anelidológicos, 12. Observaciones acerca del opérculo de Hydroides crucigera Mörch y descripción de un caso de duplicidad de este órgano. Anales del Instituto de Biología de la Universidad Nacional Autónoma de México, 15: 409-414.

Rouse, G. W., 2000. Family Serpulidae. In: Beesley, P. L., Ross, G. J. B. \& Glasby, C. J. (eds). Polychaetes \& Allies: The Southern Synthesis. Fauna of Australia. Vol. 4A. Polychaeta, Myzostomida, Pogonophora, Echiura, Sipuncula. CSIRO. Melbourne: 184-189.

Rouse, G. W. \& Fauchald, K., 1997. Cladistics and polychaetes. Zoologica Scripta, 26: 139-204. https://doi. org/10.1111/j.1463-6409.1997.tb00412.x

Rouse, G. W. \& Pleijel, F., 2001. Polychaetes. Oxford University Press. Hong Kong. 354 pp.

San Martín, G. en preparación. Familia Serpulidae. En: Ramos, M. A. et al. (eds.). Fauna Ibérica. Museo Nacional de Ciencias Naturales. CSIC. Madrid.

Sentz, E., 1962. Étude morphologique des Serpulidae Hydroides norvegica (Gunnerus) et Serpula concharum Langerhans et de leurs forms intermédiares. Vie et Milieu, 13(3): 441-452.

Zibrowius, H., 1968. Étude morphologique, systématique et écologique des Serpulidae (Annelida Polychaeta) de la région de Marseille. Recueil des Traveaux de la Station Marine d'Endoume, Bulletin, 43: 81-252.

Zibrowius, H., 1971. Les espèces méditerranéennes du genre Hydroides (Polychaeta Serpulidae). Remarques sur le prétendu polymorphisme de Hydroides uncinata. Téthys, 2(3): 691-745. 
Apéndice.-Listado de las muestras recolectadas durante las campañas oceanográficas de Fauna Ibérica (I, II, III y IV), junto con sus respectivos datos de profundidad (en metros), localidad y coordenadas geográficas donde han sido halladas.

Appendix.-List of samples collected during the oceanographic campaigns of Iberian Fauna (I, II, III and IV), along with their respective depth data (in meters), location and geographic coordinates where they have been found.

\begin{tabular}{|c|c|c|c|}
\hline Profundidad & Localidad & Coordenadas geográficas (UTM) & $\begin{array}{l}\text { Campaña y código } \\
\text { de muestra }\end{array}$ \\
\hline $24 \mathrm{~m}$ & Trafalgar & $36^{\circ} 09.81^{\prime}-36^{\circ} 10.97^{\prime} N-6^{\circ} 09.21^{\prime}-6^{\circ} 09.55^{\prime} O$ & F. I. I (56A) \\
\hline 151-152 m & Coruña & $43^{\circ} 35.58^{\prime}-43^{\circ} 35.53^{\prime} N-8^{\circ} 34.28^{\prime}-8^{\circ} 31.99^{\prime} O$ & F. I. II (101A) \\
\hline 408-435 m & Coruña & $43^{\circ} 54.48^{\prime}-43^{\circ} 55.68^{\prime} N-8^{\circ} 37.99^{\prime}-8^{\circ} 39.07^{\prime} O$ & F. I. II (102A) \\
\hline $174-180 \mathrm{~m}$ & Foz & $43^{\circ} 58.75^{\prime}-43^{\circ} 58.18^{\prime} N-7^{\circ} 13.12^{\prime}-7^{\circ} 10.48^{\prime} O$ & F. I. II (105A) \\
\hline 158-167 m & N. de Foz & $43^{\circ} 55.81^{\prime}-43^{\circ} 53.91^{\prime} N-7^{\circ} 09.01^{\prime}-7^{\circ} 07.74^{\prime} O$ & F. I. II (106A) \\
\hline 114-116 m & N. Ribadeo & $43^{\circ} 40.59^{\prime}-43^{\circ} 40.25^{\prime} N-7^{\circ} 04.35^{\prime}-7^{\circ} 02.77^{\prime} \mathrm{O}$ & F. I. II (107A) \\
\hline $142-145 m$ & N. Ribadeo & $43^{\circ} 39.04^{\prime}-43^{\circ} 50.83^{\prime} N-6^{\circ} 59.38^{\prime}-6^{\circ} 59.58^{\prime} O$ & F. I. II (109A) \\
\hline 176-200 m & NO. Cabo de Peñas & $43^{\circ} 47.72^{\prime}-43^{\circ} 48.60^{\prime} N-6^{\circ} 05.96^{\prime}-6^{\circ} 04.20^{\prime} \mathrm{O}$ & F. I. II $(111 \mathrm{~A})$ \\
\hline $116-120 \mathrm{~m}$ & N. Gijón & $43^{\circ} 43.57^{\prime}-43^{\circ} 43.85^{\prime} N-5^{\circ} 55.67^{\prime}-5^{\circ} 56.76^{\prime} O$ & F. I. II (113A) \\
\hline 72-74 m & N. Gijón & $43^{\circ} 37.71^{\prime}-43^{\circ} 38.36^{\prime} N-5^{\circ} 37.59^{\prime}-5^{\circ} 39.78^{\prime} O$ & F. I. II (114A) \\
\hline 46-48 m & E. Cabo Lastres & $43^{\circ} 31.57^{\prime}-43^{\circ} 32.26^{\prime} N-5^{\circ} 13.08^{\prime}-5^{\circ} 14.14^{\prime} O$ & F. I. II (119A) \\
\hline $130 \mathrm{~m}$ & N. Cabo Mayor & $43^{\circ} 35.83^{\prime}-43^{\circ} 35.71^{\prime} N-3^{\circ} 44.32^{\prime}-3^{\circ} 44.45^{\prime} O$ & F. I. II (127A) \\
\hline $39-40 \mathrm{~m}$ & N. Cabo Mayor & $43^{\circ} 30.91^{\prime}-43^{\circ} 30.83^{\prime} N-3^{\circ} 47.26^{\prime}-3^{\circ} 46.13^{\prime} O$ & F. I. II (134A) \\
\hline 110-108 m & N. Cabo de Ajo & $43^{\circ} 34.78^{\prime}-43^{\circ} 35.46^{\prime} N-3^{\circ} 38.33^{\prime}-3^{\circ} 38.86^{\prime} O$ & F. I. II (136A) \\
\hline $36-46 \mathrm{~m}$ & O. Punta del Pescador & $43^{\circ} 28.04^{\prime}-43^{\circ} 28.91^{\prime} N-3^{\circ} 24.16^{\prime}-3^{\circ} 24.40^{\prime} O$ & F. I. II (142A) \\
\hline $57-68 \mathrm{~m}$ & NO. Punta del Pescador & $43^{\circ} 29.91^{\prime}-43^{\circ} 30.49^{\prime} N-3^{\circ} 24.45^{\prime}-3^{\circ} 24.50^{\prime} O$ & F. I. II (143A) \\
\hline $88 \mathrm{~m}$ & NO. Cabo Villano & $43^{\circ} 28.62^{\prime}-43^{\circ} 28.64^{\prime} N-2^{\circ} 53.77^{\prime}-2^{\circ} 53.36^{\prime} O$ & F. I. II (145A) \\
\hline $135-143 m$ & N. San Sebastian & $43^{\circ} 29.56^{\prime}-43^{\circ} 29.88^{\prime} N-2^{\circ} 01.58^{\prime}-2^{\circ} 00.20^{\prime} O$ & F. I. II (148A) \\
\hline 93-101 m & N. Zumaya & $43^{\circ} 23.18^{\prime}-43^{\circ} 23.14^{\prime} N-2^{\circ} 15.00^{\prime}-2^{\circ} 15.21^{\prime} O$ & F. I. II (150A) \\
\hline $82-86 \mathrm{~m}$ & N. Cabo Santa Catalina & $43^{\circ} 25.29^{\prime}-43^{\circ} 25.09^{\prime} N-2^{\circ} 31.05^{\prime}-2^{\circ} 29.83^{\prime} O$ & F. I. II (151A) \\
\hline 104-107 m & N. de San Sebastián & $43^{\circ} 22.59^{\prime}-43^{\circ} 22.16^{\prime} \mathrm{N}-2^{\circ} 3.70^{\prime}-2^{\circ} 5.76^{\prime} \mathrm{O}$ & F. I. II (152A) \\
\hline 136-137 m & N. Cabo de Oyambre & $43^{\circ} 29.43^{\prime}-43^{\circ} 29.41^{\prime} N-4^{\circ} 20.89^{\prime}-4^{\circ} 20.68^{\prime} O$ & F. I. II (158A) \\
\hline $116-120 m$ & NO. Punta Candelaria & $43^{\circ} 45.13^{\prime}-43^{\circ} 46.53^{\prime} N-8^{\circ} 10.09^{\prime}-8^{\circ} 9.59^{\prime} O$ & F. I. II (168A) \\
\hline 158-164 m & N. Cabo Prior & $43^{\circ} 44.50^{\prime}-43^{\circ} 45.52^{\prime} N-8^{\circ} 17.68^{\prime}-8^{\circ} 18.33^{\prime} O$ & F. I. II (169A) \\
\hline 761-768 m & Banco de Galicia & $42^{\circ} 43.65^{\prime}-42^{\circ} 44.70^{\prime} N-11^{\circ} 44.99^{\prime}-11^{\circ} 43.36^{\prime} \mathrm{O}$ & F. I. II (172A) \\
\hline 769-760 m & Banco de Galicia & $42^{\circ} 42.37^{\prime}-42^{\circ} 43.00^{\prime} N-11^{\circ} 47.87^{\prime}-11^{\circ} 45.78^{\prime} \mathrm{O}$ & F. I. II (173A) \\
\hline 129-133 m & O. Cabo Finisterre & $42^{\circ} 52.85^{\prime}-42^{\circ} 50.93^{\prime} N-9^{\circ} 21.47^{\prime}-9^{\circ} 20.91^{\prime} \mathrm{O}$ & F. I. II (91A) \\
\hline $60-55 \mathrm{~m}$ & Punta d'es Murte, N. de la isla de Menorca & $40^{\circ} 04.23^{\prime}-40^{\circ} 05.20^{\prime} \mathrm{N}-4^{\circ} 11.38^{\prime}-4^{\circ} 08.00^{\prime} \mathrm{E}$ & F. I. III (213A) \\
\hline $66-53 \mathrm{~m}$ & O. Cabo Berbería, S. de la isla de Formentera & $38^{\circ} 39.27^{\prime}-38^{\circ} 36.70^{\prime} \mathrm{N}-1^{\circ} 20.47^{\prime}-1^{\circ} 20.03^{\prime} \mathrm{E}$ & F. I. III (250A) \\
\hline $3.2 \mathrm{~m}$ & N. Isla Dragonera, isla de Mallorca & $39^{\circ} 35.66^{\prime} \mathrm{N}-2^{\circ} 19.58^{\prime} \mathrm{E}$ & F. I. III (177B1) \\
\hline $31.3 \mathrm{~m}$ & N. Isla Dragonera, isla de Mallorca & $39^{\circ} 35.66^{\prime} \mathrm{N}-2^{\circ} 19.58^{\prime} \mathrm{E}$ & F. I. III (177B2) \\
\hline 86.5-86 m & N. Isla Dragonera, isla de Mallorca & $39^{\circ} 37.60^{\prime}-39^{\circ} 28.99^{\prime} \mathrm{N}-2^{\circ} 19.00^{\prime}-2^{\circ} 21.22^{\prime} \mathrm{E}$ & F. I. III (178A) \\
\hline $10 \mathrm{~m}$ & Frente a Punta Jova, O. de la isla de Mallorca & $39^{\circ} 38.30^{\prime} \mathrm{N}-2^{\circ} 25.13^{\prime} \mathrm{E}$ & F. I. III (179B2) \\
\hline $9 \mathrm{~m}$ & Frente a Punta Jova, O. de la isla de Mallorca & $39^{\circ} 38.50^{\prime} \mathrm{N}-2^{\circ} 25.13^{\prime} \mathrm{E}$ & F. I. III (179B3) \\
\hline 74-72 m & $\begin{array}{l}\text { Frente a Punta de la Foradada, NO. de la isla de } \\
\text { Mallorca }\end{array}$ & $39^{\circ} 44.33^{\prime}-39^{\circ} 45.80^{\prime} \mathrm{N}-2^{\circ} 31.86^{\prime}-2^{\circ} 33.51^{\prime} \mathrm{E}$ & F. I. III (182A) \\
\hline $22.2 \mathrm{~m}$ & Punta de la Foradada, NO. de la isla de Mallorca & $39^{\circ} 45.55^{\prime} \mathrm{N}-2^{\circ} 37.23^{\prime} \mathrm{E}$ & F. I. III (183B1) \\
\hline $21.5 \mathrm{~m}$ & Punta de la Foradada, NO. de la isla de Mallorca & $39^{\circ} 45.55^{\prime} \mathrm{N}-2^{\circ} 37.23^{\prime} \mathrm{E}$ & F. I. III (183B2) \\
\hline $13.8 \mathrm{~m}$ & Punta de la Foradada, NO. de la isla de Mallorca & $39^{\circ} 45.55^{\prime} \mathrm{N}-2^{\circ} 37.23^{\prime} \mathrm{E}$ & F. I. III (183B5) \\
\hline $21 \mathrm{~m}$ & Punta de la Foradada, NO. de la isla de Mallorca & $39^{\circ} 45.55^{\prime} \mathrm{N}-2^{\circ} 37.50^{\prime} \mathrm{E}$ & F. I. III (185B1) \\
\hline $24 \mathrm{~m}$ & Punta de la Foradada, NO. de la isla de Mallorca & $39^{\circ} 45.55^{\prime} \mathrm{N}-2^{\circ} 37.50^{\prime} \mathrm{E}$ & F. I. III (185B2) \\
\hline 33-47 m & Bahía de Pollença, N. de la isla de Mallorca & $39^{\circ} 54.76^{\prime}-39^{\circ} 55.92^{\prime} \mathrm{N}-3^{\circ} 09.20^{\prime}-3^{\circ} 12.13^{\prime} \mathrm{E}$ & F. I. III (189A) \\
\hline 56-59 m & E. Cabo del Pinar, N. de la isla de Mallorca & $39^{\circ} 52.48^{\prime}-39^{\circ} 52.60^{\prime} \mathrm{N}-3^{\circ} 13.65^{\prime}-3^{\circ} 16.68^{\prime} \mathrm{E}$ & F. I. III (192A) \\
\hline $58-59 \mathrm{~m}$ & NE. Cabo de Pera, isla de Mallorca & $39^{\circ} 46.09^{\prime}-39^{\circ} 44.17^{\prime} \mathrm{N}-3^{\circ} 32.04^{\prime}-3^{\circ} 32.35^{\prime} \mathrm{E}$ & F. I. III (194A) \\
\hline
\end{tabular}


Apéndice-(continuación).

\begin{tabular}{|c|c|c|c|}
\hline Profundidad & Localidad & Coordenadas geográficas (UTM) & $\begin{array}{l}\text { Campaña y código } \\
\text { de muestra }\end{array}$ \\
\hline $10-12 \mathrm{~m}$ & $\begin{array}{l}\text { Cueva Catedral, N. Cabo del Freu, isla de } \\
\text { Mallorca }\end{array}$ & $39^{\circ} 44.70^{\prime}-39^{\circ} 44.70^{\prime} \mathrm{N}-3^{\circ} 27.40^{\prime}-3^{\circ} 27.40^{\prime} \mathrm{E}$ & F. I. III (195B3) \\
\hline 8-29 m & N. Cabo del Freu, isla de Mallorca & $39^{\circ} 44.93^{\prime} \mathrm{N}-3^{\circ} 27.62^{\prime} \mathrm{E}$ & F. I. III (197B) \\
\hline $76 \mathrm{~m}$ & E. Cabo de Pera, isla de Mallorca & $39^{\circ} 41.70^{\prime}-39^{\circ} 42.13^{\prime} \mathrm{N}-3^{\circ} 35.90^{\prime}-3^{\circ} 38.93^{\prime} \mathrm{E}$ & F. I. III (199P) \\
\hline $149-153 \mathrm{~m}$ & Cabo Nati, NO. de la isla de Menorca & $40^{\circ} 05.41^{\prime}-40^{\circ} 06.14^{\prime} \mathrm{N}-3^{\circ} 40.76^{\prime}-3^{\circ} 44.41^{\prime} \mathrm{E}$ & F. I. III (202A) \\
\hline $31 \mathrm{~m}$ & Cabo Nati, NO. de la isla de Menorca & $40^{\circ} 43.10^{\prime} \mathrm{N}-3^{\circ} 49.42^{\prime} \mathrm{E}$ & F. I. III (203B1) \\
\hline $119 \mathrm{~m}$ & Frente a Mahón, E. de la isla de Menorca & $39^{\circ} 50.59^{\prime}-39^{\circ} 50.10^{\prime} \mathrm{N}-4^{\circ} 24.53^{\prime}-4^{\circ} 24.60^{\prime} \mathrm{E}$ & F. I. III (216A) \\
\hline $10-23 \mathrm{~m}$ & Cabo Font, S. de la isla de Menorca & $39^{\circ} 49.40^{\prime} \mathrm{N}-4^{\circ} 12.25^{\prime} \mathrm{E}$ & F. I. III (218B) \\
\hline 83-82 m & S. Cabo Font, S. de la isla de Menorca & $39^{\circ} 47.25^{\prime}-39^{\circ} 47.99^{\prime} \mathrm{N}-4^{\circ} 14.13^{\prime}-4^{\circ} 11.97^{\prime} \mathrm{E}$ & F. I. III (219A) \\
\hline 97-92 m & $\begin{array}{l}\text { S. Punta de La Guardia, SE. de la isla de } \\
\text { Mallorca }\end{array}$ & $39^{\circ} 19.30^{\prime}-39^{\circ} 17.82^{\prime} N-3^{\circ} 17.20^{\prime}-3^{\circ} 14.16^{\prime}$ E & F. I. III (222A) \\
\hline $87-90 \mathrm{~m}$ & O. Punta Salinas, S. de la isla de Mallorca & $39^{\circ} 15.20^{\prime}-39^{\circ} 12.80^{\prime} \mathrm{N}-2^{\circ} 53.60^{\prime}-2^{\circ} 55.20^{\prime} \mathrm{E}$ & F. I. III (227A) \\
\hline 79-99 m & $\begin{array}{l}48 \text { millas al SO. de Cabo Blanco, isla de } \\
\text { Mallorca }\end{array}$ & $39^{\circ} 15.30^{\prime}-39^{\circ} 15.13^{\prime} \mathrm{N}-2^{\circ} 23.20^{\prime}-2^{\circ} 27.47^{\prime} \mathrm{E}$ & F. I. III (228A) \\
\hline $28 \mathrm{~m}$ & Islote del Toro, SO. de la isla de Mallorca & $39^{\circ} 27.74^{\prime} \mathrm{N}-2^{\circ} 28.42^{\prime} \mathrm{E}$ & F. I. III (230B1) \\
\hline $12.2 \mathrm{~m}$ & Islote del Toro, SO. de la isla de Mallorca & $39^{\circ} 27.74^{\prime} \mathrm{N}-2^{\circ} 28.42^{\prime} \mathrm{E}$ & F. I. III (230B5) \\
\hline $35-43 \mathrm{~m}$ & Islote del Toro, SO. de la isla de Mallorca & $39^{\circ} 27.74^{\prime} \mathrm{N}-2^{\circ} 28.42^{\prime} \mathrm{E}$ & F. I. III (230B18) \\
\hline 113-111 m & SO. Islote del Toro, SO. de la isla de Mallorca & $39^{\circ} 24.30^{\prime}-39^{\circ} 24.41^{\prime} \mathrm{N}-2^{\circ} 24.30^{\prime}-2^{\circ} 20.73^{\prime} \mathrm{E}$ & F. I. III (231A) \\
\hline 134-137 m & NE. de la isla de lbiza & $39^{\circ} 11.40^{\prime}-39^{\circ} 10.70^{\prime} \mathrm{N}-1^{\circ} 35.20^{\prime}-1^{\circ} 39.01^{\prime} \mathrm{E}$ & F. I. III (235A) \\
\hline $17.3 \mathrm{~m}$ & Al S. del faro de la isla de Tagomago, isla de lbiza & $39^{\circ} 01.80^{\prime} \mathrm{N}-1^{\circ} 39.14^{\prime} \mathrm{E}$ & F. I. III (236B4) \\
\hline $55-56 \mathrm{~m}$ & E. Cabo Martinet, E. de la isla de lbiza & $38^{\circ} 54.07^{\prime}-38^{\circ} 56.29^{\prime} \mathrm{N}-1^{\circ} 31.14^{\prime}-1^{\circ} 32.77^{\prime} \mathrm{E}$ & F. I. III (238A) \\
\hline 3-35 m & Punta Galera, E. de la isla Espardell, isla de lbiza & $38^{\circ} 47.90^{\prime} \mathrm{N}-1^{\circ} 28.95^{\prime} \mathrm{E}$ & F. I. III (240B) \\
\hline $35 \mathrm{~m}$ & Punta Galera, E. de la isla Espardell, isla de lbiza & $38^{\circ} 47.90^{\prime} \mathrm{N}-1^{\circ} 28.95^{\prime} \mathrm{E}$ & F. I. III (240B4) \\
\hline 3-6 m & Punta Galera, E. de la isla Espardell, isla de lbiza & $38^{\circ} 47.90^{\prime} \mathrm{N}-1^{\circ} 28.95^{\prime} \mathrm{E}$ & F. I. III (240B6) \\
\hline $32-35 \mathrm{~m}$ & Punta Galera, E. de la isla Espardell, isla de lbiza & $38^{\circ} 47.90^{\prime} \mathrm{N}-1^{\circ} 28.95^{\prime} \mathrm{E}$ & F. I. III (240B10) \\
\hline 140-144 m & $\begin{array}{l}\text { Banco de Emile Baudot, SE. de la islas Islas } \\
\text { Baleares }\end{array}$ & $38^{\circ} 43.03^{\prime}-38^{\circ} 43.47^{\prime} N-2^{\circ} 29.51^{\prime}-2^{\circ} 30.81^{\prime} E$ & F. I. III (245A) \\
\hline 134-126 m & 12 millas al E. de la isla de Formentera & $38^{\circ} 45.60^{\prime}-38^{\circ} 43.60^{\prime} N-1^{\circ} 51.40^{\prime}-1^{\circ} 50.01^{\prime} \mathrm{E}$ & F. I. III (248A) \\
\hline 0-3 m & Estany des Peix, N. de la isla de Formentera & $38^{\circ} 43.46^{\prime}-38^{\circ} 43.46^{\prime} N-1^{\circ} 24.65^{\prime}-1^{\circ} 24.65^{\prime} E$ & F. I. III (254B) \\
\hline 6-45 m & N. de la isla Bleda Mayor, O. de la isla de lbiza & $38^{\circ} 58.32^{\prime}-38^{\circ} 58.32^{\prime} N-1^{\circ} 09.90^{\prime}-1^{\circ} 09.90^{\prime} \mathrm{E}$ & F. I. III (258B3) \\
\hline $10 \mathrm{~m}$ & Punta E. de Cala Eubarca, NO. de la isla de lbiza & $39^{\circ} 04.52^{\prime} \mathrm{N}-1^{\circ} 23.13^{\prime} \mathrm{E}$ & F. I. III (262B2) \\
\hline $44 \mathrm{~m}$ & Punta O. de Cala Eubarca, NO. de la isla de lbiza & $39^{\circ} 04.40^{\prime} \mathrm{N}-1^{\circ} 21.71^{\prime} \mathrm{E}$ & F. I. III (263B2) \\
\hline $113-110 \mathrm{~m}$ & $\begin{array}{l}9.8 \text { millas al S. del Placer de la Barra Alta, islas } \\
\text { Columbretes }\end{array}$ & $39^{\circ} 40.61^{\prime}-39^{\circ} 40.44^{\prime} \mathrm{N}-0^{\circ} 28.40^{\prime}-0^{\circ} 30.85^{\prime} \mathrm{E}$ & F. I. III (266A) \\
\hline 0-32 m & Placer de la Barra Alta, islas Columbretes & $39^{\circ} 49.84^{\prime} \mathrm{N}-0^{\circ} 32.32^{\prime} \mathrm{E}$ & F. I. III (267B) \\
\hline $32 \mathrm{~m}$ & Placer de la Barra Alta, islas Columbretes & $39^{\circ} 49.84^{\prime} \mathrm{N}-0^{\circ} 32.32^{\prime} \mathrm{E}$ & F. I. III (267B2) \\
\hline $32 \mathrm{~m}$ & Placer de la Barra Alta, islas Columbretes & $39^{\circ} 49.84^{\prime}-39^{\circ} 49.84^{\prime} \mathrm{N}-0^{\circ} 32.32^{\prime}-0^{\circ} 32.32^{\prime} \mathrm{E}$ & F. I. III (267B3) \\
\hline $32 \mathrm{~m}$ & Placer de la Barra Alta, islas Columbretes & $39^{\circ} 49.84^{\prime}-39^{\circ} 49.84^{\prime} \mathrm{N}-0^{\circ} 32.32^{\prime}-0^{\circ} 32.32^{\prime} \mathrm{E}$ & F. I. III (267B4) \\
\hline 86-82 m & O. de la islas Columbretes & $39^{\circ} 49.65^{\prime}-39^{\circ} 52.56^{\prime} \mathrm{N}-0^{\circ} 37.57^{\prime}-0^{\circ} 37.59^{\prime} \mathrm{E}$ & F. I. III (268A) \\
\hline $55-62 \mathrm{~m}$ & $\begin{array}{l}\text { O. de la isla Horadada-La Ferrera, islas } \\
\text { Columbretes }\end{array}$ & $39^{\circ} 52.84^{\prime}-39^{\circ} 54.07^{\prime} \mathrm{N}-0^{\circ} 39.56^{\prime}-0^{\circ} 39.14^{\prime} \mathrm{E}$ & F. I. III (269A) \\
\hline 3-26 m & O. de la isla Navarrete, islas Columbretes & $39^{\circ} 53.30^{\prime} \mathrm{N}-0^{\circ} 39.90^{\prime} \mathrm{E}$ & F. I. III (270B4) \\
\hline $18-23 \mathrm{~m}$ & Cabo del Pinar, N. de la isla de Mallorca & $39^{\circ} 53.00^{\prime} \mathrm{N}-03^{\circ} 11.80^{\prime} \mathrm{E}$ & F. I. III (191B5) \\
\hline $85-92 \mathrm{~m}$ & Canal de la isla de Menorca & $39^{\circ} 52.30^{\prime}-39^{\circ} 55.24^{\prime} \mathrm{N}-03^{\circ} 36.65^{\prime}-03^{\circ} 37.02^{\prime} \mathrm{E}$ & F. I. III (200A) \\
\hline $60 \mathrm{~m}$ & $\begin{array}{l}\text { Cara SO. del Islote Imperial, S. de la isla de } \\
\text { Cabrera }\end{array}$ & $39^{\circ} 07.55^{\prime} \mathrm{N}-2^{\circ} 57.70^{\prime} \mathrm{E}$ & F. I. III (223B1) \\
\hline $17 \mathrm{~m}$ & $\begin{array}{l}\text { Cara SO. del Islote Imperial, S. de la isla de } \\
\text { Cabrera }\end{array}$ & $39^{\circ} 07.55^{\prime} \mathrm{N}-2^{\circ} 57.70^{\prime} \mathrm{E}$ & F. I. III (223B7) \\
\hline $30 \mathrm{~m}$ & Islote del Toro, SO. de la isla de Mallorca & $39^{\circ} 27.74^{\prime} \mathrm{N}-2^{\circ} 28.42^{\prime} \mathrm{E}$ & F. I. III (230B14) \\
\hline $58-57 \mathrm{~m}$ & S. de la isla Espardell, isla de lbiza & $38^{\circ} 42.71^{\prime}-38^{\circ} 44.90^{\prime} \mathrm{N}-01^{\circ} 32.28^{\prime}-01^{\circ} 30.51^{\prime} \mathrm{E}$ & F. I. III (239A) \\
\hline 92-91 m & NO. de la isla de Formentera & $38^{\circ} 47.57^{\prime}-38^{\circ} 45.17^{\prime} \mathrm{N}-1^{\circ} 20.10^{\prime}-1^{\circ} 18.97^{\prime} \mathrm{E}$ & F. I. III (252A) \\
\hline
\end{tabular}


Apéndice-(continuación).

\begin{tabular}{|c|c|c|c|}
\hline Profundidad & Localidad & Coordenadas geográficas (UTM) & $\begin{array}{l}\text { Campaña y código } \\
\text { de muestra }\end{array}$ \\
\hline $15 \mathrm{~m}$ & N. Bahía de Pollença, isla de Mallorca & $39^{\circ} 56.50^{\prime} \mathrm{N}-3^{\circ} 11.83^{\prime} \mathrm{E}$ & F. I. III (190B5) \\
\hline $17 \mathrm{~m}$ & $\begin{array}{l}\text { Pared S. de Columbrete Grande, cerca de la } \\
\text { "Escala de España", islas Columbretes }\end{array}$ & $39^{\circ} 53.56^{\prime}-39^{\circ} 53.59^{\prime} \mathrm{N}-00^{\circ} 41.04^{\prime}-00^{\circ} 41.07^{\prime} \mathrm{E}$ & F. I. IV (274B5) \\
\hline $35 \mathrm{~m}$ & Bocana de Puerto Tofiño, islas Columbretes & $39^{\circ} 53.88^{\prime}-39^{\circ} 53.73^{\prime} \mathrm{N}-00^{\circ} 41.33^{\prime}-00^{\circ} 41.26^{\prime} \mathrm{E}$ & F. I. IV (275B1) \\
\hline $14 \mathrm{~m}$ & Bocana de Puerto Tofiño, islas Columbretes & $39^{\circ} 53.88^{\prime}-39^{\circ} 53.73^{\prime} \mathrm{N}-00^{\circ} 41.33^{\prime}-00^{\circ} 41.26^{\prime} \mathrm{E}$ & F. I. IV (275B13) \\
\hline $59.5-61 \mathrm{~m}$ & $\begin{array}{l}\text { A } 1.16 \text { millas al O. de Columbrete Grande, islas } \\
\text { Columbretes }\end{array}$ & $39^{\circ} 53.40^{\prime}-39^{\circ} 54.70^{\prime} \mathrm{N}-00^{\circ} 39.30^{\prime}-00^{\circ} 39.36^{\prime} \mathrm{E}$ & F. I. IV (276A) \\
\hline $25 \mathrm{~m}$ & Bocana de Puerto Tofiño, islas Columbretes & $39^{\circ} 53.88^{\prime}-39^{\circ} 53.73^{\prime} \mathrm{N}-00^{\circ} 41.33^{\prime}-00^{\circ} 41.26^{\prime} \mathrm{E}$ & F. I. IV (275B9) \\
\hline $7.5 \mathrm{~m}$ & Cara E. del Banco El Fidalgo, islas Columbretes & $39^{\circ} 52.78^{\prime}-39^{\circ} 52.72^{\prime} \mathrm{N}-00^{\circ} 40.09^{\prime}-00^{\circ} 40.06^{\prime} \mathrm{E}$ & F. I. IV (277B19) \\
\hline $7 \mathrm{~m}$ & Cara E. del Banco El Fidalgo, islas Columbretes & $39^{\circ} 52.78^{\prime}-39^{\circ} 52.72^{\prime} \mathrm{N}-00^{\circ} 40.09^{\prime}-00^{\circ} 40.06^{\prime} \mathrm{E}$ & F. I. IV (277B24) \\
\hline $19 \mathrm{~m}$ & Al S. de La Horadada, islas Columbretes & $39^{\circ} 52.38^{\prime}-39^{\circ} 52.36^{\prime} \mathrm{N}-00^{\circ} 40.07^{\prime}-00^{\circ} 40.28^{\prime} \mathrm{E}$ & F. I. IV (281B11) \\
\hline $19 \mathrm{~m}$ & Al S. de La Horadada, islas Columbretes & $39^{\circ} 52.38^{\prime}-39^{\circ} 52.36^{\prime} \mathrm{N}-00^{\circ} 40.07^{\prime}-00^{\circ} 40.28^{\prime} \mathrm{E}$ & F. I. IV (281B14) \\
\hline $21.5 \mathrm{~m}$ & Al S. de La Horadada, islas Columbretes & $39^{\circ} 52.38^{\prime}-39^{\circ} 52.36^{\prime} \mathrm{N}-00^{\circ} 40.07^{\prime}-00^{\circ} 40.28^{\prime} \mathrm{E}$ & F. I. IV (281B3) \\
\hline $10 \mathrm{~m}$ & Al S. de La Horadada, islas Columbretes & $39^{\circ} 52.38^{\prime}-39^{\circ} 52.36^{\prime} \mathrm{N}-00^{\circ} 40.07^{\prime}-00^{\circ} 40.28^{\prime} \mathrm{E}$ & F. I. IV (281B4) \\
\hline 3-40 m & Cara E. del Banco El Fidalgo, islas Columbretes & $39^{\circ} 52.78^{\prime}-39^{\circ} 52.72^{\prime} \mathrm{N}-00^{\circ} 40.09^{\prime}-00^{\circ} 40.06^{\prime} \mathrm{E}$ & F. I. IV (277B15) \\
\hline $20 \mathrm{~m}$ & Bocana de Puerto Tofiño, islas Columbretes & $39^{\circ} 53.88^{\prime}-39^{\circ} 53.73^{\prime} \mathrm{N}-00^{\circ} 41.33^{\prime}-00^{\circ} 41.26^{\prime} \mathrm{E}$ & F. I. IV (275B2) \\
\hline $15 \mathrm{~m}$ & Cara E. de la Piedra Joaquín, islas Columbretes & $39^{\circ} 52.68^{\prime}-39^{\circ} 52.68^{\prime} \mathrm{N}-00^{\circ} 40.23^{\prime}-00^{\circ} 40.16^{\prime} \mathrm{E}$ & F. I. IV (282B15) \\
\hline 129-132 m & N. de Ondarroa & $43^{\circ} 27.24^{\prime}-43^{\circ} 27.77^{\prime} \mathrm{N}-2^{\circ} 23.42^{\prime}-2^{\circ} 24.81^{\prime} \mathrm{O}$ & F. I. II (153A) \\
\hline 6-20 m & SO. de la isla del Aire, SO. de la isla de Menorca & $39^{\circ} 47.80^{\prime}-39^{\circ} 47.80^{\prime} \mathrm{N}-4^{\circ} 17.40^{\prime}-4^{\circ} 17.40^{\prime} \mathrm{E}$ & F. I. III (217B14) \\
\hline $15-10 \mathrm{~m}$ & Cabo Font, S. de la isla de Menorca & $39^{\circ} 49.40^{\prime} \mathrm{N}-4^{\circ} 12.25^{\prime} \mathrm{E}$ & F. I. III (218B10) \\
\hline 80- $85 \mathrm{~m}$ & $\begin{array}{l}1.12 \text { millas al SO. de La Horadada, islas } \\
\text { Columbretes }\end{array}$ & $39^{\circ} 53.09^{\prime}-39^{\circ} 51.57^{\prime} \mathrm{N}-00^{\circ} 36.80^{\prime}-00^{\circ} 39.25^{\prime} \mathrm{E}$ & F. I. IV (283A) \\
\hline $15 \mathrm{~m}$ & $\begin{array}{l}\text { Banco Díaz, N. del Islote Churruca, islas } \\
\text { Columbretes }\end{array}$ & $39^{\circ} 51.27^{\prime}-39^{\circ} 51.27^{\prime} \mathrm{N}-00^{\circ} 40.55^{\prime}-00^{\circ} 40.60^{\prime} \mathrm{E}$ & F. I. IV (285B12) \\
\hline $30 \mathrm{~m}$ & $\begin{array}{l}\text { Banco Díaz, N. del Islote Churruca, islas } \\
\text { Columbretes }\end{array}$ & $39^{\circ} 51.27^{\prime}-39^{\circ} 51.27^{\prime} \mathrm{N}-00^{\circ} 40.55^{\prime}-00^{\circ} 40.60^{\prime} \mathrm{E}$ & F. I. IV (285B22) \\
\hline $12.5 \mathrm{~m}$ & Al S. del Islote Baleato, islas Columbretes & $39^{\circ} 50.86^{\prime}-39^{\circ} 50.94^{\prime} \mathrm{N}-00^{\circ} 40.60^{\prime}-00^{\circ} 40.74^{\prime} \mathrm{E}$ & F. I. IV (290B9) \\
\hline $\mathbf{0 m}$ & $\begin{array}{l}0.29 \text { millas al NO. de El Bergantín, islas } \\
\text { Columbretes }\end{array}$ & $39^{\circ} 51.32^{\prime} \mathrm{N}-00^{\circ} 40.07^{\prime} \mathrm{E}$ & F. I. IV (291P) \\
\hline 78.5-81 m & $\begin{array}{l}1.1 \text { millas al E. de La Horadada, islas } \\
\text { Columbretes }\end{array}$ & $39^{\circ} 51.20^{\prime}-39^{\circ} 53.50^{\prime} \mathrm{N}-00^{\circ} 41.40^{\prime}-00^{\circ} 42.00^{\prime} \mathrm{E}$ & F. I. IV (293A) \\
\hline $43 \mathrm{~m}$ & Al N. de Columbrete Grande, islas Columbretes & $39^{\circ} 54.02^{\prime}-39^{\circ} 53.98^{\prime} \mathrm{N}-00^{\circ} 41.08^{\prime}-00^{\circ} 41.11^{\prime} \mathrm{E}$ & F. I. IV (295B1) \\
\hline $15 \mathrm{~m}$ & Placer de la Barra Alta, islas Columbretes & $39^{\circ} 49.77^{\prime}-39^{\circ} 49.82^{\prime} \mathrm{N}-00^{\circ} 32.10^{\prime}-00^{\circ} 32.15^{\prime} \mathrm{E}$ & F. I. IV (296B10) \\
\hline $33 \mathrm{~m}$ & Faro de la isla de Alborán al $359^{\circ}$ y a 0.66 millas & $35^{\circ} 57.04^{\prime}-35^{\circ} 57.02^{\prime} \mathrm{N}-03^{\circ} 02.09^{\prime}-03^{\circ} 02.08^{\prime} \mathrm{O}$ & F. I. IV (302B5) \\
\hline 28.7-34.7 m & Faro de la isla de Alborán al $142^{\circ}$ y a 0.54 millas & $35^{\circ} 55.99^{\prime}-35^{\circ} 55.94^{\prime} \mathrm{N}-03^{\circ} 01.57^{\prime}-03^{\circ} 01.68^{\prime} \mathrm{O}$ & F. I. IV (304A) \\
\hline 33-49 m & Faro de la isla de Alborán al $213^{\circ}$ y a 0.88 millas & $35^{\circ} 55.68^{\prime}-35^{\circ} 55.63^{\prime} \mathrm{N}-03^{\circ} 03.25^{\prime}-03^{\circ} 02.67^{\prime} \mathrm{O}$ & F. I. IV (305A) \\
\hline 37-39 m & Faro de la isla de Alborán al $145^{\circ}$ y a 0.74 millas & $35^{\circ} 55.59^{\prime}-35^{\circ} 55.77^{\prime} \mathrm{N}-03^{\circ} 02.34^{\prime}-03^{\circ} 01.54^{\prime} \mathrm{O}$ & F. I. IV (307A) \\
\hline $9.7-12.5 \mathrm{~m}$ & Faro de la isla de Alborán al $67^{\circ}$ y a 0.31 millas & $35^{\circ} 56.52^{\prime}-35^{\circ} 56.41^{\prime} \mathrm{N}-03^{\circ} 01.72^{\prime}-03^{\circ} 01.72^{\prime} \mathrm{O}$ & F. I. IV (309B) \\
\hline $15 \mathrm{~m}$ & $\begin{array}{l}\text { Faro de la isla de Alborán al } 70.05^{\circ} \text { y a } 0.39 \\
\text { millas }\end{array}$ & $35^{\circ} 56.50^{\prime}-35^{\circ} 56.50^{\prime} \mathrm{N}-03^{\circ} 01.62^{\prime}-03^{\circ} 01.62^{\prime} \mathrm{O}$ & F. I. IV (311B3) \\
\hline $187-118 \mathrm{~m}$ & $\begin{array}{l}\text { Dorsal de la isla de Alborán. Faro al } 238^{\circ} \text { y a } \\
12.95 \text { millas }\end{array}$ & $35^{\circ} 49.54^{\prime}-35^{\circ} 49.14^{\prime} \mathrm{N}-03^{\circ} 16.04^{\prime}-03^{\circ} 17.15^{\prime} \mathrm{O}$ & F. I. IV (312A) \\
\hline $118 \mathrm{~m}$ & $\begin{array}{l}\text { Dorsal de la isla de Alborán. Faro al } 238^{\circ} \text { y a } \\
11.3 \text { millas }\end{array}$ & $35^{\circ} 49.91^{\prime}-35^{\circ} 50.36^{\prime} \mathrm{N}-03^{\circ} 14.63^{\prime}-03^{\circ} 13.72^{\prime} \mathrm{O}$ & F. I. IV (313A) \\
\hline $0.5-99 \mathrm{~m}$ & $\begin{array}{l}\text { Faro al } 235^{\circ} \text { y a } 12.1 \text { millas. Dorsal de la isla de } \\
\text { Alborán }\end{array}$ & $35^{\circ} 49.77^{\prime}-35^{\circ} 49.50^{\prime} \mathrm{N}-03^{\circ} 15.05^{\prime}-03^{\circ} 14.80^{\prime} \mathrm{O}$ & F. I. IV (314A) \\
\hline $36 \mathrm{~m}$ & $\begin{array}{l}\text { Faro al } 63.75^{\circ} \text { y a } 3.18 \text { millas. Dorsal de la isla } \\
\text { de Alborán }\end{array}$ & $35^{\circ} 58.00^{\prime}-35^{\circ} 58.00^{\prime} \mathrm{N}-02^{\circ} 58.46^{\prime}-02^{\circ} 58.46^{\prime} \mathrm{O}$ & F. I. IV (315B2) \\
\hline $90-240 \mathrm{~m}$ & $\begin{array}{l}\text { Faro al } 237^{\circ} \text { y a } 12.15 \text { millas. Dorsal de la isla de } \\
\text { Alborán }\end{array}$ & $35^{\circ} 50.03^{\prime}-35^{\circ} 49.77^{\prime} \mathrm{N}-03^{\circ} 15.23^{\prime}-03^{\circ} 14.45^{\prime} \mathrm{O}$ & F. I. IV (316A) \\
\hline 87-213 m & $\begin{array}{l}\text { Faro al } 229^{\circ} \text { y a } 6.3 \text { millas. Dorsal de la isla de } \\
\text { Alborán }\end{array}$ & $35^{\circ} 52.49^{\prime}-35^{\circ} 52.31^{\prime} \mathrm{N}-03^{\circ} 08.90^{\prime}-03^{\circ} 07.92^{\prime} \mathrm{O}$ & F. I. IV (317A) \\
\hline
\end{tabular}


Apéndice-(continuación)

\begin{tabular}{|c|c|c|c|}
\hline Profundidad & Localidad & Coordenadas geográficas (UTM) & $\begin{array}{l}\text { Campaña y código } \\
\text { de muestra }\end{array}$ \\
\hline 95-99 m & $\begin{array}{l}\text { Faro al } 8.5^{\circ} \text { y a } 2.55 \text { millas. Dorsal de la isla de } \\
\text { Alborán }\end{array}$ & $35^{\circ} 58.84^{\prime}-35^{\circ} 58.88^{\prime} \mathrm{N}-03^{\circ} 00.18^{\prime}-03^{\circ} 01.66^{\prime} \mathrm{O}$ & F. I. IV (322A) \\
\hline 67-78.7 m & $\begin{array}{l}\text { Faro al } 33.5^{\circ} \text { y a } 1.4 \text { millas. Dorsal de la isla de } \\
\text { Alborán }\end{array}$ & $35^{\circ} 57.82^{\prime}-35^{\circ} 57.50^{\prime} \mathrm{N}-03^{\circ} 00.04^{\prime}-03^{\circ} 01.16^{\prime} \mathrm{O}$ & F. I. IV (323A) \\
\hline $51-58 \mathrm{~m}$ & Faro de la isla de Alborán al $4^{\circ}$ y a 0.81 millas & $35^{\circ} 57.17^{\prime}-35^{\circ} 57.18^{\prime} \mathrm{N}-03^{\circ} 02.00^{\prime}-03^{\circ} 03.00^{\prime} \mathrm{O}$ & F. I. IV (324A) \\
\hline 415-463 m & $\begin{array}{l}\text { Faro al } 229^{\circ} \text { y a } 8.25 \text { millas. Dorsal de la isla de } \\
\text { Alborán }\end{array}$ & $35^{\circ} 52.71^{\prime}-35^{\circ} 52.16^{\prime} \mathrm{N}-03^{\circ} 12.90^{\prime}-03^{\circ} 10.67^{\prime} \mathrm{O}$ & F. I. IV (326A) \\
\hline 115-125 m & $\begin{array}{l}\text { Faro al } 188^{\circ} \text { y a } 3.3 \text { millas. Dorsal de la isla de } \\
\text { Alborán }\end{array}$ & $35^{\circ} 53.05^{\prime}-35^{\circ} 53.01^{\prime} \mathrm{N}-03^{\circ} 02.59^{\prime}-03^{\circ} 02.48^{\prime} \mathrm{O}$ & F. I. IV (327A) \\
\hline $40 \mathrm{~m}$ & $\begin{array}{l}\text { Faro al } 62.5^{\circ} \text { y a } 3.16 \text { millas. Dorsal de la isla de } \\
\text { Alborán }\end{array}$ & $35^{\circ} 57.80^{\prime} \mathrm{N}-02^{\circ} 58.61^{\prime} \mathrm{O}$ & F. I. IV (328B2) \\
\hline$?$ & N. de la isla Bleda Mayor, O. de la isla de lbiza. & $38^{\circ} 58.32^{\prime} \mathrm{N}-1^{\circ} 09.90^{\prime} \mathrm{E}$ & F. I. III (258B10) \\
\hline
\end{tabular}

